\title{
Plant nutrition for sustainable development and global health
}

\author{
Johan Six
}

Received: 14 November 2010 / Accepted: 22 November 2010 / Published online: 7 December 2010

(C) The Author(s) 2010. This article is published with open access at Springerlink.com

Plant nutrition is a fundamental science that impacts all aspects of cropping systems, environmental sustainability, and human health and well being. Therefore, there is a need for increased understanding of the fundamental principles of plant nutrition, which can help develop and extend optimized field practices and improve public policies to ensure sustainable food production. This Special Issue addresses this need and is derived from studies presented at the XVI International Plant Nutrition Colloquium (IPNC), "Plant Nutrition for Sustainable Development and Global Health". The publications compiled in this Special Issue focus on elucidating the effects of agricultural management on the interactions between soil and plant to efficiently provide nutrients to plants and to ensure a sustained food production with minimal environmental impact.

In two papers, Janssen illustrates the pertinent need for more long-term agricultural experiments in Africa. In both studies, Janssen re-analyzes data from longterm experiments to elucidate the causes of success or failure with regard to sustained soil productivity. In his first paper, he employs a simple model to two of the very few existing long-term experiments in Kenya. In the follow-up paper, Janssen uses the

Responsible Editor: Hans Lambers.

J. Six $(\bowtie)$

Department of Plant Sciences, University of California,

Davis, CA 95616, USA

e-mail: jwsix@ucdavis.edu concept of crop nutrient equivalents (CNE) on two NPK field trials, one in Vietnam and one in the Netherlands.

In the next paper, Vanlauwe et al. provide a comprehensive evaluation of the Integrated Soil Fertility Management (ISFM) approach, an approach that has been increasingly adopted by the research and development community as a framework for boosting crop productivity with minimal environmental impacts. The ISFM approach relies on four components: proper fertilizer management, use of improved varieties, combined application of organic inputs and fertilizer, and adaptation of input application rates to within-farm soil fertility gradients. In their paper, Vanlauwe et al. rigorously evaluate, through metaanalysis, the impact of these different components on the agronomic efficiency of fertilizer in maize cropping systems in Sub-Saharan Africa.

In recent years, biofuel systems have become a more prevalent feature within the agricultural landscape and the efficient use of $\mathrm{N}$ for minimal environmental impact is as critical in these systems as in traditional food production systems. Both Thorburn et al. and Guretzky et al. address fertilizer management practices in biofuel systems (i.e. sugarcane and switchgrass, respectively).

While much research in plant nutrition has focused on $\mathrm{N}$, other nutrients also play a crucial role in sustainable food production and security. First of all, $\mathrm{P}$ deficiency is a major limiting factor for plant growth worldwide and plant species have developed a 
wide diversity of adaptive strategies in response to $\mathrm{P}$ limitations. As in many other studies, Yang et al. focus on the genetic variation in P-use efficiency. More specifically, they map the quantitative trait loci (QTL) for P efficiency from a cross between Brassica napus P-inefficient cv. B104-2 and P-efficient cv. Eyou Changjia. George et al., in contrast, emphasize the rarely considered robustness of genetic variation in contrasting management conditions. They compare the P-nutrition of winter and spring barley genotypes in a field trial with different tillage practices (conventional plough vs. minimum tillage). Secondly, it is now apparent that the historical focus in fertilization and breeding strategies for macronutrients, such as $\mathrm{N}$, $\mathrm{P}$, and $\mathrm{K}$, has resulted in an inadvertent decline in the micronutrient content of many staple food crops. In their paper, Zuo \& Zhang summarize recent progress in soil and crop management to resolve complex plant $\mathrm{Fe}$ nutritional problems through manipulating the rhizosphere and improving crop management. Hammac et al. present and test a novel approach to in situ imaging of the rhizosphere, which allows the observation of species differences in root-hair development in response to water availability. Their method should be useful in future studies of rhizosphere interactions and crop water and nutrient management.

In the last paper of this Special Issue, He et al. evaluate the integrated total $\mathrm{N}$ input (ITNI) system, which is a method to quantify total atmospheric $\mathrm{N}$ deposition. More specifically, they determined the effect of pot surface area and variety of indicator plant on the amount of airborne $\mathrm{N}$ input quantified by the ITNI system.

The 2009 IPNC was co-sponsored by the University of California-Davis, The Department of Plant Sciences at UC Davis, PotashCorp, the International Plant Nutrition Institute, Brandt, Springer, and Oxford University Press. I want to thank the sponsors for making the colloquium possible and all participants that made it a success.

Open Access This article is distributed under the terms of the Creative Commons Attribution Noncommercial License which permits any noncommercial use, distribution, and reproduction in any medium, provided the original author(s) and source are credited. 